\title{
The economic and clinical burden of early versus late initiation of celecoxib among patients with osteoarthritis
}

This article was published in the following Dove Press journal:

ClinicoEconomics and Outcomes Research

\author{
Ahmed Shelbaya ${ }^{1,2}$ \\ Caitlyn T Solem ${ }^{3}$ \\ Chris Walker ${ }^{4}$ \\ Yin Wan ${ }^{3}$ \\ Courtney Johnson ${ }^{3}$ \\ Joseph C Cappelleri'
}

'Pfizer Inc., New York, NY, ${ }^{2}$ Columbia School of Public Health, New York, NY, ${ }^{3}$ Pharmerit International, Bethesda, MD, USA; ${ }^{4}$ ffizer Ltd., Tadworth, Surrey, UK
Correspondence: Caitlyn T Solem Pharmerit International, 4350 East-West Highway, Suite I I I0, Bethesda, MD 208I4, USA

$\mathrm{Tel}+|24082| 1267$

Fax +12408211296

Email csolem@pharmerit.com
Objective: This study aimed to evaluate the characteristics associated with early versus late initiation of celecoxib treatment after osteoarthritis (OA) diagnosis and whether economic and safety outcomes differ between patients with early versus late initiation of celecoxib.

Methods: Adults ( $\geq 18$ years) with a confirmed OA diagnosis (International Classification of Diseases, 9th Edition, Clinical Modifications code: 715.XX), $\geq 12$ months of continuous pre- and post-index enrollment, and $\geq 1$ post-index claim for celecoxib were included from the MarketScan ${ }^{\circledR}$ Commercial Claims and Encounter Database (2009-2013). Index date was defined as initial OA diagnosis. Patients were categorized as initiating celecoxib early (within 6 months of index date) or late ( $\geq 6$ months after index date). Logistic regressions were used to assess characteristics associated with early versus late celecoxib initiation. Key outcomes included health care resource utilization (HCRU) and costs post-index, and adverse event incidence post-celecoxib initiation. Unadjusted and adjusted comparisons (using generalized linear models with a gamma distribution for costs and Poisson distribution for event and resource utilization) were made between early and late celecoxib initiators.

Results: Of the 62,434 OA patients identified, 27,402 were early and 35,032 were late initiators. Post-index hospital admissions and length of stay did not differ statistically between early versus late initiators after controlling for pre-index event rates and covariates, but early patients had significantly fewer outpatient (incidence rate ratio [IRR]: 0.96; 95\% confidence interval [CI]: 0.95, 0.97) and emergency room visits (IRR: 0.89; 95\% CI: 0.84, 0.95). After adjustment for key covariates, early initiators (versus late initiators) had lower all-cause (US\$12,909 versus US\$13,781, $P<0.001$ ) and OA-related (US $\$ 4,988$ versus US $\$ 5,178, P=0.015$ ) costs per person-year. Early initiators had no statistically significant difference in the incidence of post-celecoxib cardiovascular (IRR: $0.92 ; 95 \%$ CI: 0.73, 1.14), gastrointestinal (IRR: 1.25; 95\% CI: 0.81, 1.92), or renal (IRR: 1.19; 95\% CI: 0.65, 2.18) events, controlling for pre-index event rates and covariates when compared to late initiators. Conclusion: In this real-world cohort, patients initiated on celecoxib early (versus late) had significantly lower costs and HCRU; this may warrant consideration when making treatment decisions for OA patients.

Keywords: osteoarthritis, celecoxib, nonsteroidal anti-inflammatory drug, economic burden, health care resource use

\section{Introduction}

Osteoarthritis (OA), which is estimated to affect 30.8 million adults in the US, or $13.4 \%$ of the US population, is the most common joint disorder in the US and is expected to become more prevalent as the population ages. ${ }^{1-3}$ The disease is characterized by loss of articular cartilage, bone spur formation, and localized inflammation, which can cause 
the patient to experience swelling, pain, stiffness, and reduced range of motion of the affected joints..$^{2,4-6}$ The knee, hip, and hand are most affected by the disease. ${ }^{1,2,6}$ Common risk factors for OA include obesity, sedentary lifestyle, injury, and overuse of affected joint..$^{7-9}$ Usually, OA develops progressively as joint damage increases over time, which can potentially decrease the quality of life and lead to fatigue, mental health problems, loss of work productivity, and possible disability. ${ }^{4-7,10}$

Treatment guidelines for OA suggest patients first try non-pharmacologic interventions and then pharmacologic interventions. The Osteoarthritis Research Society International (OARSI), American College of Rheumatology (ACR), European League Against Rheumatism (EULAR), and National Institute for Health and Care Excellence (NICE) suggest that if able, patients try physical therapy, exercise, or weight loss (if the patient is obese or overweight) in the case of knee or hip OA. For pharmacologic intervention, guidelines suggest physicians prescribe acetaminophen, dependent on comorbidities and concomitant medication. If acetaminophen does not provide adequate relief, guidelines suggest physicians prescribe nonsteroidal anti-inflammatory drugs (NSAIDs) including cyclooxygenase-2 (COX-2) inhibitors. While both non-selective and COX-2 selective NSAIDs can cause serious side effects, patients in the latter group must be monitored closely for gastrointestinal, renal, and cardiovascular adverse events (AEs). ${ }^{4,11-14}$

Celecoxib (Celebrex ${ }^{\circledR}$, Pfizer, Inc., New York, NY, USA) is an NSAID that selectively inhibits COX-2, an enzyme responsible for induction of pain. ${ }^{15}$ It is approved by the US Food and Drug Administration and indicated for the relief of various chronic musculoskeletal conditions including signs and symptoms of OA. ${ }^{16}$ Celecoxib has been shown to be as effective as non-selective NSAIDs like naproxen and diclofenac, yet associated with fewer gastrointestinal side effects. . $^{9,15,17,18}$

To our knowledge, no studies have assessed the effects of starting OA medications earlier versus later, with respect to diagnosis, on outcomes. There are potential benefits of diagnosing and treating arthritis early; however, no studies provide supporting evidence in OA. The objectives of this study were, therefore, to describe treatment patterns in terms of time to start celecoxib and patient characteristics associated with early treatment, and subsequently evaluate the effect of initiating celecoxib early versus late among patients diagnosed with OA.

\section{Methods \\ Data source}

This was a retrospective cohort study using data from the 2009 to 2013 Truven MarketScan Commercial and Medicare Supple- mental Database (MarketScan). MarketScan is a commercial claims database that contains patient-level demographic, diagnosis, inpatient, outpatient, procedure, prescription (via Red Book), ${ }^{19}$ and payment information for millions of US patients with private and public health insurance. The longitudinal nature of this database allows patient care to be tracked from diagnosis to end of treatment, for multiple health care encounters. ${ }^{19}$ Since the statistical analyses in this study utilized deidentified secondary data, institutional review board approval was not required.

\section{Study population}

Adult patients with an OA diagnosis (International Classification of Diseases, 9th Edition, Clinical Modifications [ICD-9-CM] code 715.XX) were obtained from MarketScan. The patient's initial OA diagnosis (inpatient or outpatient) observed during the study period was defined as the index date (Figure 1). To be included, patients had to be 18 years or older and have had continuous enrollment for at least 12 months before and after index date (Figure 2).

\section{Medication exposure}

Celecoxib was identified in Red Book by searching for "celecoxib" in the generic drug name category, reflecting the only selective COX-2 inhibitor product available from 2009 to 2013. Time from index date until first prescription of celecoxib was dichotomized to define early and late celecoxib initiators. Early initiators were patients who filled their first prescription for celecoxib within the first 6 months of their index date. Late initiators were patients who filled their first prescription for celecoxib 6 months or more after their index date. Six months was chosen as the separator between early and late celecoxib initiation because a previous study by Essex et al identified 6 months as the maximum duration over which the efficacy of celecoxib has been assessed in a clinical trial for OA. ${ }^{9}$ Additionally, database analyses of OA patients in the US and UK found that the majority of patients switched or discontinued OA treatment between 6 months and 1 year of treatment initiation. ${ }^{20,21}$

Proton pump inhibitors (PPIs) and pain medications were identified in Red Book. Pre-index and concomitant medication use (medication claim occurring between the first and last prescription fill for celecoxib) were identified, and dichotomized as any use, during the respective periods.

\section{Study outcomes}

The primary outcomes of this study included health care resource utilization (HCRU) and associated costs. Incidences of all-cause and OA-related HCRU were measured 12 months 


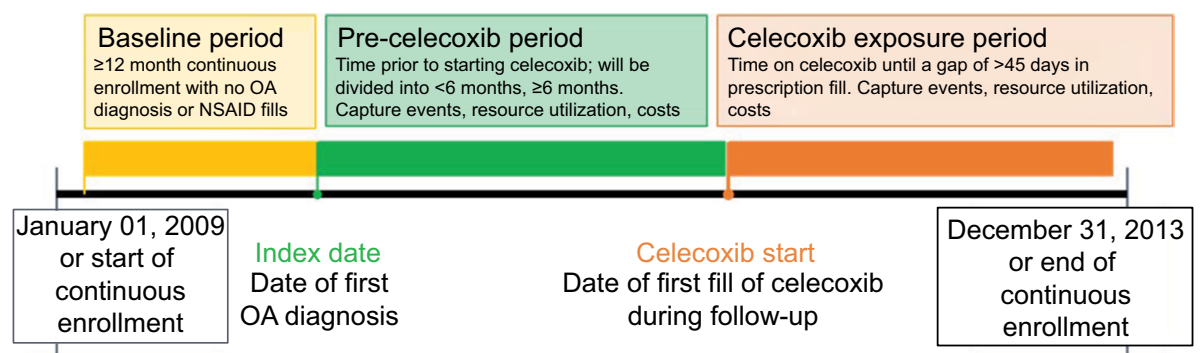

Continuous enrollment

Figure I Study design of time to initiation of celecoxib.

Abbreviations: OA, osteoarthritis; NSAID, nonsteroidal anti-inflammatory drugs.

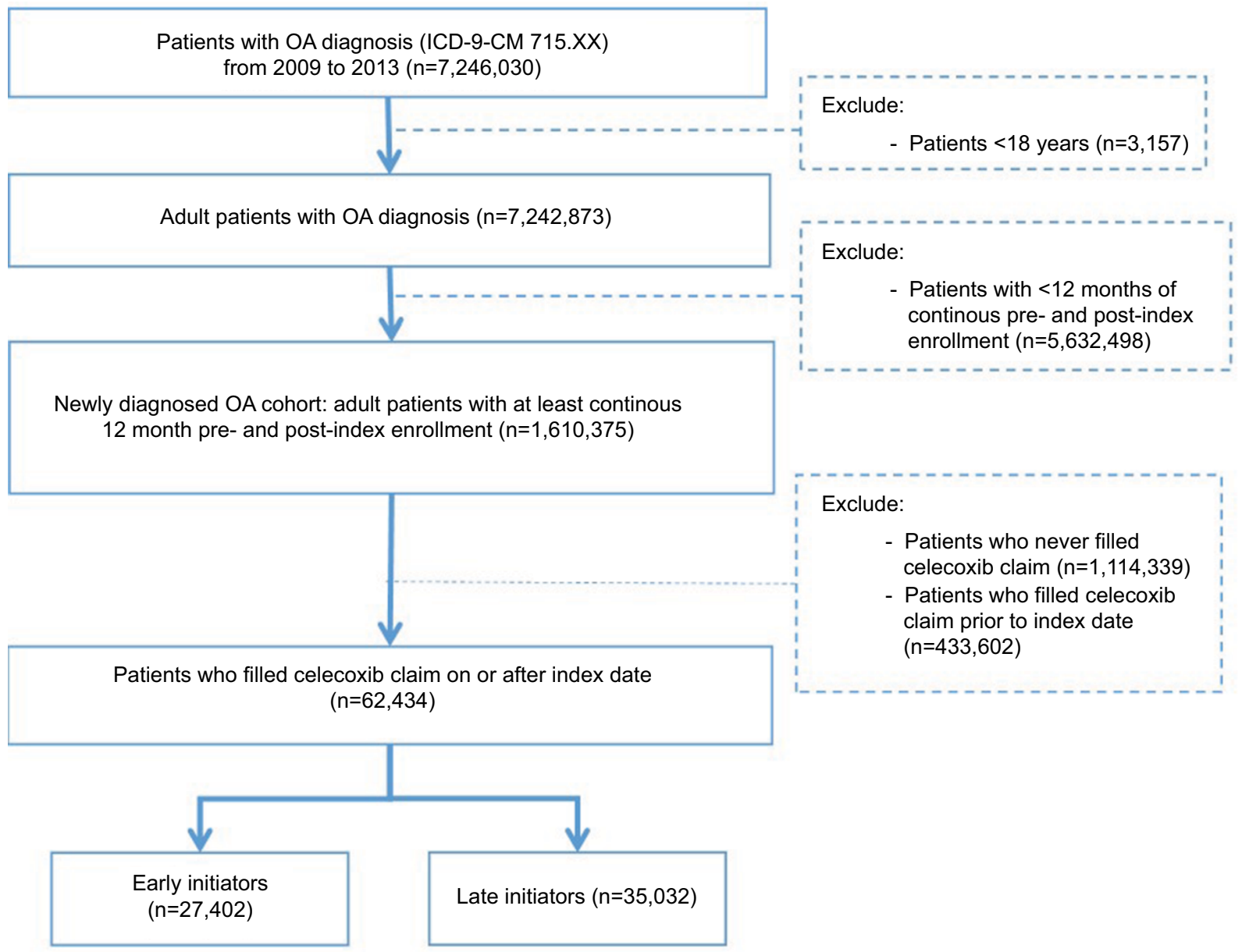

Figure 2 Flowchart for cohort selection

Abbreviations: OA, osteoarthritis; ICD-9-CM, International Classification of Diseases, 9th Edition, Clinical Modifications.

before index date and during the follow-up period. The recorded incidences were subsequently compared between early and late initiators. To be considered OA-related, the HCRU and cost claim had to have an associated ICD-9-CM code of 715.XX. Hospital admissions, length of stay, emergency room (ER) visits, and outpatient visits were all factors included in determining HCRU. Outpatient visits included office, independent clinic, state public health clinic, and rural health clinic visits. All-cause and OA-related costs were similarly computed for all patients and compared between early and late celecoxib initiators. Costs included inpatient, outpatient, ER, and drug costs. All costs were inflated to 2014 US dollars.

Additional outcomes included incidence of key gastrointestinal, cardiovascular, and renal events. Incidence of events 
of interest that occurred during the follow-up period, stratified into time pre- and post-celecoxib initiation were compared between early and late celecoxib initiators. Events of interest included gastrointestinal bleeds (ICD-9-CM code: 578.9) and gastrointestinal perforation (ICD-9-CM codes: 569.83, 863.9), which were grouped into gastrointestinal events; myocardial infarction (MI; ICD-9-CM codes: 410-410.9, 412); and congestive heart failure (CHF; ICD-9-CM code: 428.0), grouped into cardiovascular events; and chronic nephritis (ICD-9-CM codes: 582-582.9), nephritis and nephropathy (ICD-9-CM codes: 583-583.7), chronic kidney disease (ICD-9-CM codes: 585-585.9), renal failure (ICD-9-CM code: 586), disorders resulting from impaired renal function (ICD-9-CM codes: 588-588.9), grouped into renal events. To be considered unique, gastrointestinal events must have occurred 7 days apart, ${ }^{22}$ while cardiovascular events must have occurred 56 days apart to ensure it was not a readmission for the same cardiovascular event. ${ }^{23}$

\section{Statistical analyses}

Unless otherwise stated, categorical statistics were described as frequency and proportion of the patient cohort, while continuous statistics were summarized as mean and standard deviation. After descriptively comparing characteristics between early and late initiators, multivariable logistic regression was used to find characteristics associated with early versus late celecoxib initiation. ${ }^{24}$ The predetermined covariates used in the regression analysis included age, sex, payer, region, pre-index events measured during 12 month baseline period, pre-index use of PPIs, pain medication, and Charlson comorbidity index (CCI).

Unadjusted incidence of all-cause HCRU per person-year (including hospital admissions, length of stay, ER visits, and outpatient visits) was calculated as the total number of events divided by the total number of person-years follow-up within each OA cohort; the corresponding confidence intervals were then calculated using a Poisson distribution. ${ }^{25}$ The incidence rate ratio of HCRU in the post-index period, controlling for pre-index HCRU rates, were calculated using a Poisson regression, ${ }^{26,27}$ adjusting for gender, age, pre-index events measured during 12 month baseline period, pre-index use of PPIs, and pre-index use of pain medication.

Unadjusted costs post-index were summarized per person-year. Additionally, the difference between early versus late initiator costs, and corresponding 95\% confidence intervals were calculated. Adjusted costs were calculated using a generalized linear model with a gamma distribution, ${ }^{26,28}$ adjusting for age, sex, payer, region, pre-index events measured during 12 month baseline period, pre-index use of PPIs, pre-index use of pain medication, and CCI.
Unadjusted incidence rates for renal, gastrointestinal, and cardiovascular events were calculated as the number of events divided by 100 person-years of follow-up prior to and after celecoxib use. The corresponding 95\% confidence intervals were calculated using a Poisson distribution. ${ }^{25}$ The adjusted incidence rate ratio of events in the post-celecoxib period, controlling for pre-celecoxib event rates, was calculated using a Poisson regression, ${ }^{26,27}$ adjusting for gender, age, pre-index events measured during 12 month baseline period, pre-index use of PPIs, and pre-index use of pain medication.

Analysis in this paper was generated using SAS software version 9.4 and STATA software version 14.1.

\section{Results}

A total of 62,434 patients $(27,402$ early initiators; 35,032 late initiators) were eligible for inclusion. Both early and late celecoxib initiator cohorts were mostly females and had mean age of 60 and 61 years old respectively (Table 1). Early initiators experienced fewer pre-index gastrointestinal, cardiovascular, or renal events as well as lower PPI and pain medication use during the pre-index period when compared to late initiators. Additionally, early initiators experienced significantly lower pain medication use in the period from index date to celecoxib start than later initiators $(22 \%$ versus $30 \%, P<0.001)$.

\section{Medication exposure}

The mean time to initiating celecoxib from index date was 1.8 months (standard deviation [SD] 1.8) in the early cohort and 18.3 months (SD 9.3) in the late cohort. About $32 \%$ of patients started celecoxib within 3 months of index date, while 39\% started 12 months after index date (Figure 3). After adjustment for other patient characteristics, older and female patients from the west and north central US were more likely to start celecoxib late ( $P<0.001$ for both groups). Also, patients with pre-index pain medication prescriptions were more likely to start celecoxib late $(P<0.001)$ (Table 2$)$.

\section{Resource utilization and costs}

Across both early and late celecoxib initiators, hospital admissions, length of stay, and ER visits increased post-index as compared to pre-index (Table 3). Pre-index, the proportion of patients with any HCRU (hospital admission, outpatient visit, or ER visit), was similar in early and late initiators; however, late versus early initiators had significantly higher outpatient visits per person-year (9.8 [SD 9.6] versus 9.2 [SD $9.3]$ visits; $P<0.001)$. Post-index, late (versus early) initiators, had significantly higher all-cause hospital admissions, outpatient visits, and ER visits (all $P<0.001)$ and higher 
Table I Pre-index demographic and clinical characteristics

\begin{tabular}{|c|c|c|c|}
\hline Variable & $\begin{array}{l}\text { All new OA patients } \\
(N=62,434)\end{array}$ & $\begin{array}{l}\text { Early initiators of celecoxib } \\
(n=27,402)\end{array}$ & $\begin{array}{l}\text { Late initiators of celecoxib } \\
(n=35,032)\end{array}$ \\
\hline Age, mean (SD) & $61(12.2)$ & $60(12.2)$ & $61(12.0)$ \\
\hline 18-44 years & $4,729(7.6 \%)$ & $2,426(8.9 \%)$ & $2,303(6.6 \%)$ \\
\hline $45-64$ years & $37,952(60.8 \%)$ & $16,987(62.0 \%)$ & $20,965(59.8 \%)$ \\
\hline$>65$ years & $19,753(31.6 \%)$ & $7,989(29.2 \%)$ & II,764 (33.6\%) \\
\hline Sex, male, N (\%) & $24,97 \mid(40.0)$ & II,535 (42.I) & $13,436(38.4)$ \\
\hline \multicolumn{4}{|l|}{ Region, N (\%) } \\
\hline North Central & $16,818(26.9)$ & $7,305(26.7)$ & $9,513(27.2)$ \\
\hline Northeast & $8,888(14.2)$ & $3,882(14.2)$ & $5,006(14.3)$ \\
\hline South & $25,689(4 I . I)$ & I I,709 (42.7) & 13,980 (39.9) \\
\hline West & $10,140(16.2)$ & $4,230(15.4)$ & $5,910(16.9)$ \\
\hline Unknown & $899(1.4)$ & $276(1.0)$ & $623(1.8)$ \\
\hline \multicolumn{4}{|l|}{ Insurance type, N (\%) } \\
\hline POS & $5,179(8.3)$ & $2,211(8.1)$ & $2,968(8.5)$ \\
\hline $\mathrm{HMO}$ & $5,949(9.5)$ & $2,382(8.7)$ & $3,567(10.2)$ \\
\hline PPO & $33,344(53.4)$ & I5,027 (54.8) & $18,317(52.3)$ \\
\hline Comprehensive & $12,750(20.4)$ & $5,395(19.7)$ & $7,355(21.0)$ \\
\hline Other & $5,212(8.3)$ & $2,387(8.7)$ & $2,825(8.1)$ \\
\hline \multicolumn{4}{|c|}{ Charlson-Deyo comorbidity index, N (\%) } \\
\hline 0 & $38,394(61.5)$ & $17,127(62.5)$ & $21,267(60.7)$ \\
\hline 1 & $12,483(20.0)$ & $5,413(19.8)$ & $7,070(20.2)$ \\
\hline 2 & $6,905(11.1)$ & $2,924(10.7)$ & $3,981(11.4)$ \\
\hline$\geq 3$ & $4,652(7.5)$ & $1,938(7.1)$ & $2,714(7.7)$ \\
\hline Mean (SD) & $0.7(1.2)$ & $0.7(1.2)$ & $0.7(1.3)$ \\
\hline \multicolumn{4}{|l|}{ PPI use, ${ }^{b} \mathrm{~N}(\%)$} \\
\hline Pre-index & $15,269(24.5)$ & $6,487(23.7)$ & $8,782(25.1)$ \\
\hline \multicolumn{4}{|l|}{ Pain medication, ${ }^{\mathrm{c}} \mathrm{N}(\%)$} \\
\hline Pre-index & $39,454(63.2)$ & $16,892(61.6)$ & $22,562(64.4)$ \\
\hline Post-index pre-celecoxib & $16,665(26.7)$ & $6,128(22.4)$ & $10,537(30.1)$ \\
\hline \multicolumn{4}{|c|}{ Clinical events prior to index, $\mathrm{N}(\%)$} \\
\hline Gastrointestinal $^{d}$ & $10,757(17.2)$ & $4,548(16.6)$ & $6,209(17.7)$ \\
\hline Cardiovasculare & $31,237(50.0)$ & 13,446 (49.1) & $|7,79|(50.8)$ \\
\hline Renalf $^{f}$ & $1,932(3.1)$ & $805(2.9)$ & $1,127(3.2)$ \\
\hline
\end{tabular}

Notes: aThe "other" insurance category includes exclusive provider organization, consumer-driven health plan, high deductible health plan.

'Rabeprazole, pantoprazole, esomeprazole, dexlansoprazole, lansoprazole. 'NSAID, narcotic analgesics, non-narcotic analgesics, salicylates analgesics, coformulated: NSAID and opioid, NSAID and PPI, NSAID and $\mathrm{H} 2$ blockers. ${ }^{\mathrm{O}}$ Gastric, peptic, and duodenal ulcers, dyspepsia, gastrointestinal bleeding and perforations, inflammatory bowel disease, gastroesophageal reflux disease, diarrhea, constipation. ${ }^{\circ}$ Congestive heart failure, myocardial infarction, coronary heart disease, hypertension, peripheral vascular disease, pulmonary embolism, cerebrovascular disease (stroke and transient ischemic attack). 'Acute and chronic renal disease, insufficiency, and failure.

Abbreviations: OA, osteoarthritis; POS, point of service; HMO, health maintenance organization; PPO, preferred provider organization; PPI, proton pump inhibitor; NSAID, nonsteroidal anti-inflammatory drug.

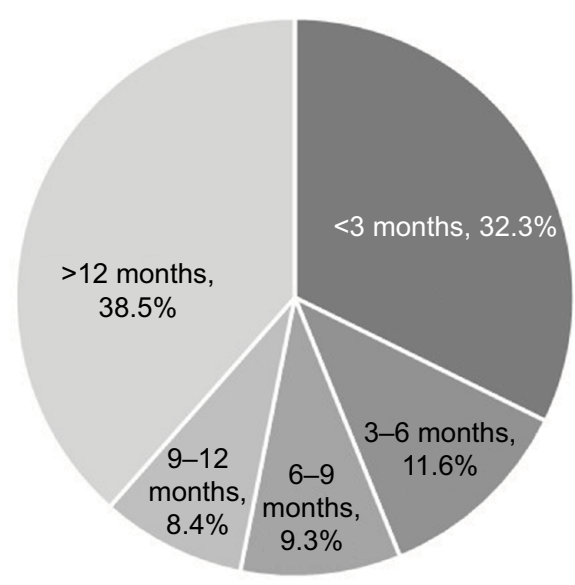

Figure 3 Time since diagnosis of $O A$ until initiation of celecoxib. Abbreviation: OA, osteoarthritis.
OA-related hospital admissions $(P=0.007)$ and outpatient physician visits $(P<0.001)$. Most all-cause and OA-related HCRU was due to outpatient visits. For all-cause outpatient visits, early initiators had 14.8 (SD 11.9) visits per personyear, whereas late initiators had 16.5 visits (SD 12.2) per person-year. For OA-related outpatient visits, early initiators had 2.1 (SD 3.4) visits per person-year and late initiators had 2.4 visits (SD 3.4) per person-year. Early (versus late) celecoxib initiators had significantly fewer outpatient (IRR: 0.96; 95\% CI: $0.95,0.97$ ) and ER visits (IRR: 0.89; 95\% CI: $0.84,0.95$ ) per person-year in the post-index period after controlling for pre-index resource use and covariates. Hospital admissions and length of stay were similar between groups (Table 3). 
Table 2 Multivariate logistic regression of patient characteristics associated with late versus early celecoxib initiation

\begin{tabular}{|c|c|c|c|}
\hline Variable & Category & Odds ratio $(95 \% \mathrm{Cl})$ & $P$-value \\
\hline \multirow[t]{3}{*}{ Age } & 18-44 years & Reference & \\
\hline & $45-64$ years & $1.30(1.23,1.39)$ & $<0.001$ \\
\hline & $\geq 65$ years & $1.62(1.5 \mathrm{I}, \mathrm{I} .73)$ & $<0.001$ \\
\hline \multirow[t]{5}{*}{ Region } & South & Reference & \\
\hline & North Central & $1.07(1.03,1.12)$ & $<0.001$ \\
\hline & Northeast & $1.05(1.00,1.10)$ & 0.061 \\
\hline & West & $1.10(1.05,1.15)$ & $<0.001$ \\
\hline & Unknown & $1.14(0.88,1.48)$ & 0.332 \\
\hline \multirow[t]{5}{*}{ Insurance type } & PPO & Reference & \\
\hline & POS & $1.09(1.03,1.15)$ & 0.004 \\
\hline & $\mathrm{HMO}$ & $1.20(1.14,1.27)$ & $<0.001$ \\
\hline & Comprehensive & $0.90(0.86,0.95)$ & $<0.001$ \\
\hline & Others or missing & $0.96(0.90,1.02)$ & 0.178 \\
\hline \multirow[t]{2}{*}{ Sex } & Male & Reference & \\
\hline & Female & $1.15(1.12,1.19)$ & $<0.001$ \\
\hline Charlson-Deyo comorbidity index & & $1.00(0.99,1.02)$ & 0.752 \\
\hline Pre-index gastrointestinal condition ${ }^{\mathrm{a}}$ & $\geq$ I claim & $1.04(0.99,1.09)$ & 0.083 \\
\hline Pre-index cardiovascular condition ${ }^{\mathrm{b}}$ & $\geq$ I claim & $1.00(0.97,1.04)$ & 0.79 \\
\hline Pre-index renal condition ${ }^{c}$ & $\geq$ I claim & $1.01(0.92,1.12)$ & 0.785 \\
\hline Pre-index PPI use ${ }^{d}$ & $\geq$ I claim & $1.02(0.98,1.07)$ & 0.232 \\
\hline Pre-index pain medication use $\mathrm{e}^{\mathrm{e}}$ & $\geq$ I claim & $1.13(1.09,1.17)$ & $<0.001$ \\
\hline
\end{tabular}

Notes: aGastric, peptic, and duodenal ulcers, dyspepsia, gastrointestinal bleeding and perforations, inflammatory bowel disease, gastroesophageal reflux disease, diarrhea, constipation. ${ }^{b}$ Congestive heart failure, myocardial infarction, coronary heart disease, hypertension, peripheral vascular disease, pulmonary embolism, cerebrovascular disease (stroke and transient ischemic attack). 'Acute and chronic renal disease, insufficiency, and failure. 'Rabeprazole, pantoprazole, esomeprazole, dexlansoprazole, lansoprazole. eNSAID, narcotic analgesics, non-narcotic analgesics, salicylates analgesics, coformulated: NSAID and opioid, NSAID and PPI, NSAID and H2 blockers.

Abbreviations: PPO, preferred provider organization; POS, point of service; HMO, health maintenance organization; PPI, proton pump inhibitor; NSAID, nonsteroidal anti-inflammatory drug.

Table 3 Incidence rates per person-year of all-cause HCRU in early versus late initiators from the pre- to post-index period

\begin{tabular}{|c|c|c|c|c|}
\hline \multirow[b]{2}{*}{ Type of HCRU } & \multicolumn{2}{|c|}{ Unadjusted incidence $(95 \% \mathrm{Cl})$} & \multirow{2}{*}{$\begin{array}{l}\text { Unadjusted } \\
\text { incidence rate ratio } \\
\text { (early versus late) }\end{array}$} & \multirow{2}{*}{$\begin{array}{l}\text { Adjusted incidence rate } \\
\text { ratio (early versus late, } \\
\text { controlling for pre-index } \\
\text { incidence) }^{\mathrm{a}}\end{array}$} \\
\hline & $\begin{array}{l}\text { Early celecoxib } \\
\text { initiators } \\
(n=27,402)\end{array}$ & $\begin{array}{l}\text { Late celecoxib } \\
\text { initiators } \\
(n=35,032)\end{array}$ & & \\
\hline \multicolumn{5}{|c|}{ Hospital admissions } \\
\hline Pre-index & $0.085(0.082,0.087)$ & $0.090(0.088,0.092)$ & $0.94(0.91,0.97)$ & - \\
\hline Post-index & $0.252(0.248,0.255)$ & $0.27 I(0.267,0.274)$ & $0.93(0.91,0.95)$ & $0.99(0.95,1.04)$ \\
\hline \multicolumn{5}{|l|}{ Length of stay } \\
\hline Pre-index & $0.342(0.337,0.346)$ & $0.350(0.346,0.354)$ & $0.98(0.96,0.99)$ & - \\
\hline Post-index & $0.942(0.935,0.950)$ & $1.001(0.995,1.007)$ & $0.94(0.93,0.95)$ & $0.97(0.90,1.5 \mathrm{I})$ \\
\hline \multicolumn{5}{|c|}{ Outpatient physician visits } \\
\hline Pre-index & 8.743 (NE, 8.765) & 9.313 (NE, 9.333) & $0.94(0.93,0.94)$ & - \\
\hline Post-index & 14.602 (NE, I4.630) & 16.214 (NE, 16.239) & $0.90(0.89,0.90)$ & $0.96(0.95,0.97)$ \\
\hline \multicolumn{5}{|c|}{ Emergency room visits } \\
\hline Pre-index & $0.075(0.073,0.077)$ & $0.083(0.08 \mathrm{I}, 0.085)$ & $0.91(0.88,0.94)$ & - \\
\hline Post-index & $0.103(0.100,0.105)$ & $0.126(0.124,0.128)$ & $0.81(0.79,0.84)$ & $0.89(0.84,0.95)$ \\
\hline
\end{tabular}

Notes: ancidence rate ratio of post-index events in early initiators as compared to the late initiators, controlling for pre-index rate of events as well as age, sex, pre-index events measured during 12 month baseline period, pre-index use of proton pump inhibitors, pain medication use, and Charlson-Deyo comorbidity index. "-" represented as data not applicable.

Abbreviations: HCRU, health care resource utilization; NE, not estimable.

Late initiators had significantly higher all-cause $(P<0.001)$ and OA-related $(P=0.015)$ costs than early initiators (Figures 4 and 5). Adjusted all-cause costs per person-year were US\$13,781 (95\% CI: US\$13,559,
US\$14,003) in late initiators and US\$12,909 (95\% CI: US\$12,673, US\$13,144) in early initiators. Adjusted OA-related costs per person-year were US\$5,178 (95\% CI: US $\$ 5,073$, US $\$ 5,283)$ in late initiators and US $\$ 4,988(95 \%$ 


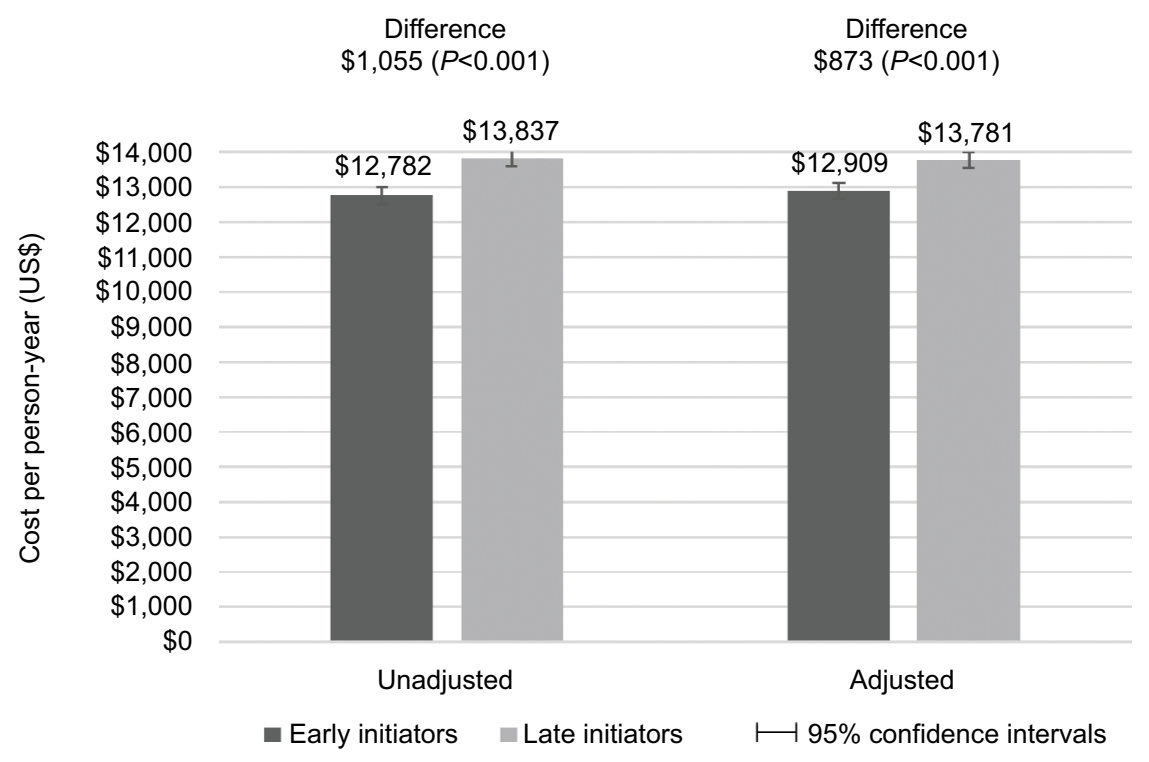

Figure 4 All-cause costs per person-year between early and late initiators.

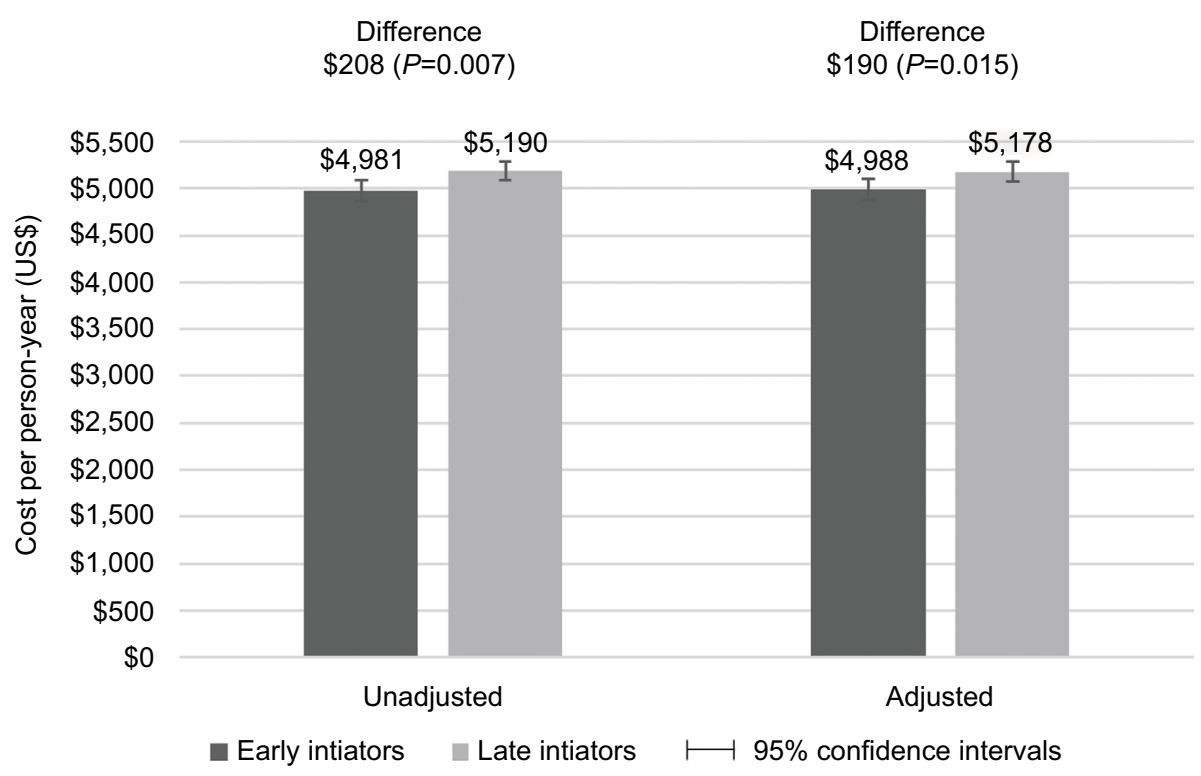

Figure 5 OA-related costs per person-year between early and late initiators

Abbreviation: $\mathrm{OA}$, osteoarthritis.

CI: US\$4,873, US\$5,102) in early initiators. Although patients had very few inpatient visits, over $50 \%$ of all-cause costs were due to inpatient claims, while almost $80 \%$ of OA-related costs were due to inpatient claims.

\section{AEs of interest}

After adjustment for differences in key covariates, late initiators tended to have lower pre- and post-celecoxib AEs than early initiators (Table 4). Early versus late initiators had a greater (although non-significant) reduction in incidence of cardiovascular clinical events (IRR: 0.92; 95\% CI 0.73, 1.14) from the pre-celecoxib to post-celecoxib period. However, early versus late initiators also had a lower (but again nonsignificant) reduction in gastrointestinal (IRR: $1.25 ; 95 \%$ CI: $0.81,1.93$ ) and renal incidence rates (IRR: $1.19 ; 95 \% \mathrm{CI}: 0.65,2.18$ ).

\section{Discussion}

This study provides valuable insight into the economic burden associated with early versus late initiation of celecoxib among OA patients. Almost half of OA patients observed initiated celecoxib within 6 months, with older age, female gender, and prescription of pain medications before OA diagnosis 
Table 4 Incidence rates per 100 person-years of gastrointestinal, cardiovascular, and renal events in early versus late initiators in the pre- to post-celecoxib period

\begin{tabular}{|c|c|c|c|c|}
\hline & \multicolumn{2}{|c|}{ Unadjusted incidence $(95 \% \mathrm{Cl})$} & \multirow{2}{*}{$\begin{array}{l}\text { Unadjusted } \\
\text { incidence rate ratio } \\
\text { (early versus late) }\end{array}$} & \multirow{2}{*}{$\begin{array}{l}\text { Adjusted incidence rate } \\
\text { ratio (early versus late, } \\
\text { controlling for pre- } \\
\text { celecoxib incidence) }^{a}\end{array}$} \\
\hline & $\begin{array}{l}\text { Early celecoxib } \\
\text { initiators } \\
(n=27,402)\end{array}$ & $\begin{array}{l}\text { Late celecoxib } \\
\text { initiators } \\
(n=35,032)\end{array}$ & & \\
\hline \multicolumn{5}{|c|}{ Gastrointestinal events } \\
\hline Pre-celecoxib & $0.67(0.45,0.98)$ & $0.46(0.40,0.52)$ & I.47 $(0.96,2.18)$ & - \\
\hline Post-celecoxib & $0.45(0.40,0.50)$ & $0.25(0.22,0.28)$ & $1.84(1.55,2.18)$ & $1.25(0.81,1.92)$ \\
\hline \multicolumn{5}{|c|}{ Cardiovascular events } \\
\hline Pre-celecoxib & $2.96(2.46,3.54)$ & $1.53(1.42,1.64)$ & $1.94(1.59,2.35)$ & - \\
\hline Post-celecoxib & $1.76(1.66,1.86)$ & $0.99(0.93,1.05)$ & $1.78(1.64,1.94)$ & $0.92(0.73,1.14)$ \\
\hline \multicolumn{5}{|l|}{ Renal disorder } \\
\hline Pre-celecoxib & $2.67(2.20,3.22)$ & $2.04(1.92,2.16)$ & $1.31(1.07,1.60)$ & - \\
\hline Post-celecoxib & $1.85(1.76,1.96)$ & $1.26(1.19,1.33)$ & I.47 (I.36, I.59) & $1.19(0.65,2.18)$ \\
\hline
\end{tabular}

Notes: ancidence rate ratio of post-celecoxib events in early initiators as compared to late initiators, controlling for pre-celecoxib rate of events and additionally adjusting for age, sex, pre-index events measured during 12 month baseline period, pre-index use of proton pump inhibitors, pain medication, Charlson-Deyo comorbidity index. "-" represented as data not applicable.

among characteristics most strongly associated with early celecoxib prescribing. The study suggests that early initiation of celecoxib was associated with lower resource use and costs compared to late initiators. Although, gastrointestinal, cardiovascular, and renal events were higher prior to initiating celecoxib and dropped after initiation in both early and late initiators, no difference in post-celecoxib event rates was found between early and late initiators after controlling for pre-celecoxib event incidence.

Appropriate disease management requires early OA diagnosis to make the most effective use of the many available treatments. Traditional pharmacologic treatments for OA have been facing increased challenges and limitations due to toxicity or loss of efficacy. Celecoxib is one of the newer agents to treat $\mathrm{OA}$ and a large number of randomized clinical trials have demonstrated its clinical efficacy, longterm safety, and generally better profile..$^{9,17,29-32}$ Celecoxib's better AE profile may explain the decrease in $\mathrm{AE}$ incidence from the pre- to post-celecoxib period in both early and late initiators, as observed in this study.

For some patients, disease management may be heavily influenced by the economic burden of the treatment; previous studies have estimated the mean per patient OA-related costs to be between US\$5,000 and US\$7,000 per year. ${ }^{5,33} \mathrm{~A}$ recent systematic review concluded that the use of celecoxib resulted in lower direct medical costs (compared to nonselective NSAIDs alone or in combination with gastroprotective agents) based on the data from 24 studies. ${ }^{34}$ Additionally, a prior analysis that examined the economic burden associated with patients who were persistent on celecoxib versus those who switched treatments using the MarketScan claims database found that celecoxib patients who switched to other treatments had significantly higher costs and health care resource use compared to those who continued to use celecoxib. ${ }^{33}$ Similar results were seen in our analysis, where patients who started celecoxib treatment later (and may have used other medications prior to celecoxib) had significantly higher costs and health care resource use than those who started treatment early. However, in the authors' understanding, no studies to date have evaluated the impact of initiating celecoxib early or late on economic and clinical outcomes using large claims database.

It is important to interpret the study results in the context of limitations. First, the medications observed in the claims database represent the prescriptions fills, which may not reflect how the patients take the medications; similarly, our analysis does not include any over-the-counter pain medication use. Second, due to the lack of clinical details from using a claims database, there may be unobserved factors confounding the study results. In particular, exercise and weight loss may affect the severity of knee or hip OA, but information regarding these lifestyle changes is not available within claims databases. Third, given the nature of the retrospective analysis using a claims database, the causal relationship between the events evaluated here and celecoxib drug use cannot be defined. For example, attribution of events could be related to other medicines or comorbidities, and not the NSAID itself.

\section{Conclusion}

In real-world settings, $43.9 \%$ of patients initiated celecoxib within 6 months of OA diagnosis. Early initiation of celecoxib (relative to OA diagnosis) was associated with lower 
economic burden compared to late initiation. This study did not find any statistically significant difference in observed post-celecoxib AEs between early versus late initiators after adjusting for pre-celecoxib event incidence; higher unadjusted pre-celecoxib event incidence in the early (versus late) initiators may suggest that this could be a driver of earlier prescribing of celecoxib. This study provides robust data and valuable information particularly on the economic outcomes associated with early as compared to late initiation of celecoxib using a large claims database.

\section{Acknowledgments}

The abstract of this paper was previously presented at the $21 \mathrm{st}$ Annual International Society for Pharmacoeconomics and Outcomes Research 2016 Congress in Washington, DC, as a poster presentation with interim findings. The poster's abstract was published in "ISPOR 21st Annual International Meeting Research Abstracts" in Value in Health: DOI 10.1016/j. jval.2016.03.1113. This study was sponsored by Pfizer.

\section{Disclosure}

Ahmed Shelbaya, Chris Walker, and Joseph C Cappelleri are employees of Pfizer. Caitlyn T Solem and Courtney Johnson are employees of Pharmerit International who were paid consultants to Pfizer in connection with the development of this manuscript. Yin Wan was an employee of Pharmerit International and paid consultant to Pfizer when the work was conducted. The authors report no other conflicts of interest in this work.

\section{References}

1. CDC. Arthritis-Related Statistics. Available from: https://www.cdc.gov/ arthritis/data_statistics/arthritis-related-stats.htm. Accessed January 25, 2018.

2. What Is Osteoarthritis? Fast Facts: An Easy-to-Read Series of Publications for the Public. 2014. Available from: https://www.niams.nih.gov/ sites/default/files/catalog/files/osteoarthritis_ff.pdf. Accessed January 25, 2018

3. Cisternas MG, Murphy L, Sacks JJ, Solomon DH, Pasta DJ, Helmick CG. Alternative methods for defining osteoarthritis and the impact on estimating prevalence in a US population-based survey. Arthritis Care Res (Hoboken). 2016;68(5):574-580.

4. NICE. Osteoarthritis: care and management. NICE clinical guideline 177. 2014. Available from: https://www.nice.org.uk/guidance/cg177/ resources/osteoarthritis-care-and-management-pdf-35109757272517. Accessed January 22, 2018.

5. Le KT, Montejano LB, Cao Z, Zhao Y, Ang D. Healthcare costs associated with osteoarthritis in US patients. Pain Pract. 2012;12(8):633-640.

6. Bijlsma JW, Berenbaum F, Lafeber FP. Osteoarthritis: an update with relevance for clinical practice. Lancet. 2011;377(9783):2115-2126.

7. Palazzo C, Nguyen C, Lefevre-Colau M-M, Rannou F, Poiraudeau S. Risk factors and burden of osteoarthritis. Ann Phys Rehabil Med. 2016;59(3):134-138.

8. Leyland KM, Judge A, Javaid MK, et al. Obesity and the relative risk of knee replacement surgery in patients with knee osteoarthritis: a prospective cohort study. Arthritis Rheumat. 2016;68(4): $817-825$.
9. Essex M, Bhadra P, Sands G. Efficacy and tolerability of celecoxib versus naproxen in pateints with osteoarthritis of the knee: a randomized, double-blind, double-dummy trial. J Int Med Res. 2012; 40(4):1357-1370.

10. Bannuru RR, Schmid CH, Kent DM, Vaysbrot EE, Wong JB, McAlindon TE. Comparative effectiveness of pharmacologic interventions for knee osteoarthritis: a systematic review and network meta-analysis. Ann Int Med. 2015;162(1):46-54.

11. Zhang W, Moskowitz RW, Nuki G, et al. OARSI recommendations for the management of hip and knee osteoarthritis, part II: OARSI evidence-based, expert consensus guidelines. Osteoarthritis Cartilage. 2008;16(2):137-162.

12. Zhang W, Doherty M, Arden N, et al. EULAR evidence based recommendations for the management of hip osteoarthritis: report of a task force of the EULAR Standing Committee for International Clinical Studies Including Therapeutics (ESCISIT). Ann Rheum Dis. 2004;64(5):669-681.

13. Jordan KM, Arden NK, Doherty M, et al. EULAR recommendations 2003: an evidence based approach to the management of knee osteoarthritis: report of a Task Force of the Standing Committee for International Clinical Studies Including Therapeutic Trials (ESCISIT). Ann Rheum Dis. 2003;62(12):1145-1155.

14. Hochberg MC, Altman RD, April KT, et al. American College of Rheumatology 2012 recommendations for the use of nonpharmacologic and pharmacologic therapies in osteoarthritis of the hand, hip, and knee. Arthritis Care Res. 2012;64(4):465-474.

15. Pairet M, Van Ryn J. COX-2 Inhibitors. Basel, Switzerland: Birkhäuser; 2012.

16. Pfizer I. CELEBREX - celecoxib capsule. 2015. Available from http:// labeling.pfizer.com/ShowLabeling.aspx?id=793. Accessed December $12,2015$.

17. Singh G, Fort JG, Goldstein JL, et al. Celecoxib versus naproxen and diclofenac in osteoarthritis patients: SUCCESS-I Study. Am J Med. 2006; 119(3):255-266.

18. Deeks JJ, Smith LA, Bradley MD. Efficacy, tolerability, and upper gastrointestinal safety of celecoxib for treatment of osteoarthritis and rheumatoid arthritis: a systematic review of randomised controlled trials. BMJ. 2002;325(7365):619.

19. Hansen LG, Chang S. White paper - health research data for the real world: the MarketScan databases. 2011. Available from: http:// truvenhealth.com/portals/0/assets/PH_11238_0612_TEMP_MarketScan_WP_FINAL.pdf. Accessed January 25, 2018.

20. Gore M, Sadosky A, Leslie D, Tai K-S, Seleznick M. Patterns of therapy switching, augmentation, and discontinuation after initiation of treatment with select medications in patients with osteoarthritis. Clin Ther. 2011;33(12):1914-1931.

21. Gore M, Sadosky AB, Leslie DL, Tai K-S, Emery P. Therapy switching, augmentation, and discontinuation in patients with osteoarthritis and chronic low back pain. Pain Pract. 2012;12(6):457-468.

22. Ghate SR, Biskupiak J, Ye X, Kwong WJ, Brixner DI. All-cause and bleeding-related health care costs in warfarin-treated patients with atrial fibrillation. J Manag Care Pharm. 2011;17(9):672-684.

23. Kiyota Y, Schneeweiss S, Glynn RJ, Cannuscio CC, Avorn J, Solomon $\mathrm{DH}$. Accuracy of medicare claims-based diagnosis of acute myocardial infarction: estimating positive predictive value on the basis of review of hospital records. Am Heart J. 2004;148(1):99-104.

24. Hosmer DW Jr, Lemeshow S, Sturdivant RX. Applied Logistic Regression. 3rd ed. Hoboken, NJ: Wiley; 2013.

25. C.I. Calculator: Single Incidence Rate. Available from: https://www2. ccrb.cuhk.edu.hk/stat/confidence $\% 20$ interval/CI\%20for $\% 20$ single $\% 20$ rate.htm. Accessed April 25, 2016.

26. Dobson AJ, Barnett A. An introduction to generalized linear models. 3rd ed. Boca Raton, FL: Chapman and Hall/CRC; 2008.

27. Meyer BD. Natural and quasi-experiments in economics. Journal of Business \& Economic Statistics. 1995;13(2):151-161.

28. Blough DK, Ramsey SD. Using Generalized Linear Models to Assess Medical Care Costs. Health Services and Outcomes Research Methodology. 2000;1(2):185-202. 
29. Becker M, Wang T, Wisniewski L, et al. Rationale, design, and governance of Prospective Randomized Evaluation of Celecoxib Integrated Safety versus Ibuprofen Or Naproxen (PRECISION), a cardiovascular end point trial of nonsteroidal antiinflammatory agents in patients with arthritis. Am Heart J. 2009;157(4):606-612.

30. Chan FKL, Lanas A, Scheiman J, Berger MF, Nguyen H, Goldstein JL. Celecoxib versus omperazole and diclofenac in patients with osteoarthritis and rheumatoid arthritis (CONDOR): a randomised trial. Lancet. 2010;376(9736):173-179.

31. Nissen SE, Yeomans ND, Solomon DH, et al. Cardiovascular safety of celecoxib, naproxen, or ibuprofen for arthritis. $N$ Engl J Med. 2016;375(26):2519-2529.
32. Zhang J, Ding EL, Song YS. Adverse effects of cyclooxygenase 2 inhibitors on renal and arrhythmia events: meta-analysis of randomized trials. JAMA. 2006;296(13):1619-1632.

33. Ji X, Liu S, Solem CT, et al. Generic switch evaluation of Celebrex ${ }^{\circledR}$ in patients with osteoarthritis (OA) using a retrospective claims database. Value Health. 2015;18(3):A158.

34. Huelin R, Pokora T, Foster TS, Mould JF. Economic outcomes for celecoxib: a systematic review of pharmacoeconomic studies. Expert Rev Pharmacoecon Outcomes Res. 2012;12(4):505-523.
ClinicoEconomics and Outcomes Research

\section{Publish your work in this journal}

ClinicoEconomics and Outcomes Research is an international, peerreviewed open-access journal focusing on health technology assessment, pharmacoeconomics and outcomes research in the areas of diagnosis, medical devices, and clinical, surgical and pharmacological intervention. The economic impact of health policy and health systems
Submit your manuscript here: https://www.dovepress.com/clinicoeconomics-and-outcomes-research-journal
Dovepress

organization also constitute important areas of coverage. The manuscript management system is completely online and includes a very quick and fair peer-review system, which is all easy to use. Visit http://www.dovepress.com/testimonials.php to read real quotes from published authors. 